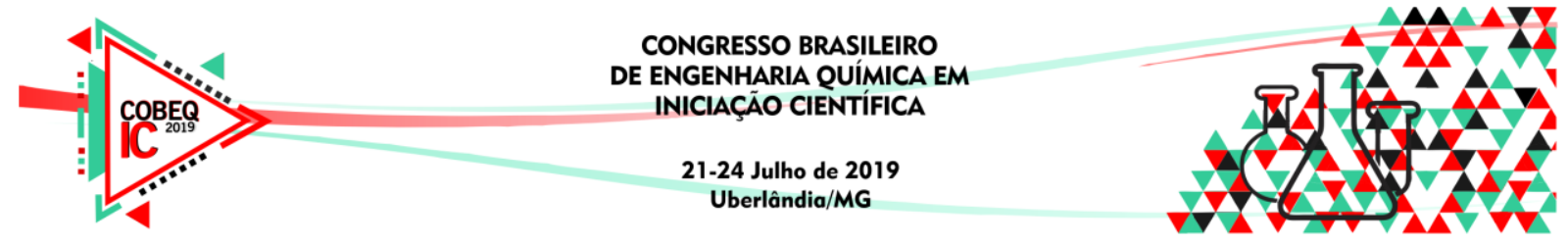

\title{
INVESTIGAÇÃO DA INFLUÊNCIA DA GEOMETRIA DE ENTRADA NA FLUIDODINÂMICA DE UM LEITO DE JORRO MODIFICADO VIA TÉCNICAS DE SIMULAÇÃO CFD
}

\author{
J. C. REZENDE ${ }^{1}$, R. S. FONTOURA ${ }^{1}$, H. B. BRAGA ${ }^{1}$, D. A. SANTOS ${ }^{1}$ \\ ${ }^{1}$ Universidade Federal de Goiás, Instituto de Química, Curso de Engenharia Química; \\ E-mail para contato: hanielbraga@ hotmail.com
}

\begin{abstract}
RESUMO - o presente trabalho objetiva investigar as influências da geometria de entrada do leito e da altura de leito estático sobre a fluidodinâmica no interior de um leito de jorro modificado via simulações CFD. Tanto com relação aos dados experimentais, quanto com relação aos resultados numéricos, a mudança da geometria de entrada do leito de jorro, da circular para a retangular (mesmo diâmetro hidráulico), levou a uma diminuição dos valores de velocidade de mínimo jorro, independente da altura de leito estático de material. O método de determinação da condição de mínimo jorro simulada através da visualização da distribuição da fração de sólidos, em detrimento da metodologia gráfica, melhor condiz com o observado experimentalmente.
\end{abstract}

\section{INTRODUÇÃO}

O leito de jorro, desenvolvido inicialmente por Mathur e Gishler (1955), é um equipamento amplamente utilizado nos processos industriais que exigem o contato efetivo entre um fluido e partículas. A aplicação deste equipamento abrange sistemas de resfriamento de sólidos, granulação, revestimento de partículas e cristalização. O movimento cíclico e uniforme das partículas no interior do leito de jorro, característico deste equipamento, proporciona um bom contato fluido-partícula, garantindo elevados coeficientes de transferência de calor e massa, tornando-o adequado em processos de secagem e inoculação de grãos (DUARTE, 2006).

Com o constante avanço na área de processamento e armazenamento de dados, a utilização das ferramentas de CFD (Computational Fluid Dynamics) vem se tornando populares. Tais técnicas aliam os conhecimentos em fenômenos de transporte de massa, movimento e energia às soluções numéricas, fazendo com que seja possível prever determinados comportamentos de fluidos e sistemas particulados nos mais diversos ambientes (SANTOS, 2011).

Dessa forma, o presente trabalho objetiva investigar as influências da geometria de entrada do leito e da altura de leito estático sobre a fluidodinâmica no interior de um leito de jorro modificado via simulações CFD. 


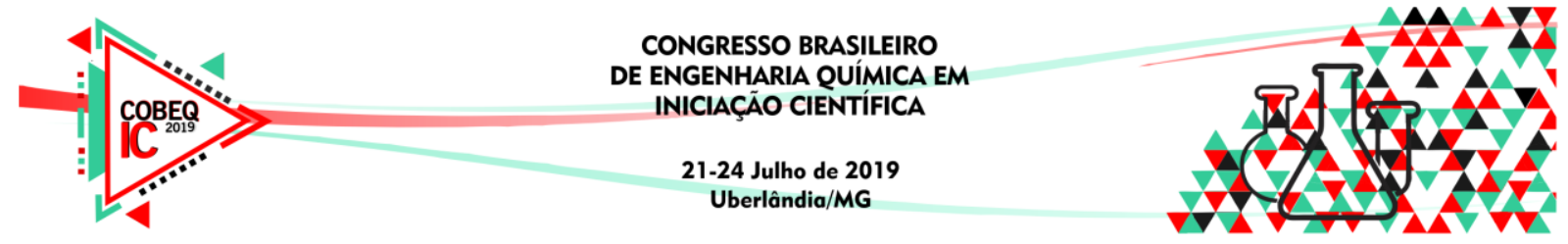

\section{MATERIAIS E METODOLOGIA}

\subsection{Metodologia Experimental}

A unidade experimental utilizada neste trabalho é composta por um leito de jorro cilíndrico modificado de acrílico com $0,048 \mathrm{~m}$ de diâmetro e $0,48 \mathrm{~m}$ de altura. $\mathrm{O}$ termo modificado se refere ao fato do leito aqui em questão não possuir uma parte cônica, mas, tão somente, uma parte cilíndrica. Este leito se diferencia de um leito fluidizado pela ausência de um distribuidor de fluido uniforme em sua base, sendo que, ao contrário, possui um único orifício de entrada. Duas diferentes geometrias de entradas foram investigadas: uma entrada circular e uma entrada retangular. O diâmetro hidráulico de ambas as entradas foi o mesmo, cujo valor é de $0,0166 \mathrm{~m}$. Foram empregadas, também neste estudo, duas diferentes alturas de leito estático de material: $0,07 \mathrm{~m}$ e $0,13 \mathrm{~m}$.

As partículas utilizadas no leito de jorro foram esferas de vidro de alta precisão com $4 \mathrm{~mm}$ de diâmetro e $2500 \mathrm{Kg} \cdot \mathrm{m}^{-3}$ de densidade. A porosidade do leito de material medida foi $\phi=0,34$. Para a fase fluida foi utilizada a água $\left(\rho=998,2 \mathrm{~kg} \cdot \mathrm{m}^{-3} \mathrm{e} \mu=0,001 \mathrm{~kg} \cdot \mathrm{m}^{-1} \cdot \mathrm{s}^{-1}\right)$.

\subsection{Metodologia Numérica}

Para a criação da geometria e malha computacional, assim como para a realização das simulações numéricas de CFD, utilizou-se do pacote ANSYS STUDENT ${ }^{\circledR} 19.0$, versão acadêmica. Com relação à modelagem do escoamento multifásico (água-sólido) presente no leito de jorro, empregou-se a modelagem Euleriana Granular Multifásica juntamente com a Teoria Cinética do Escoamento Granular. Para maiores detalhes sobre a modelagem e os métodos numéricos aqui utilizados, ver Santos (2011).

A malha computacional utilizada foi em 3D e totalmente hexaédrica. Com o intuito de avaliar o efeito do número de células ou volumes sobre os resultados numéricos obtidos, foi realizado um teste de independência de malhas. Desta forma, empregou-se, para todas as simulações, uma malha computacional contendo 207.000 células ou volumes, em que não se observou mais variações significativas nos resultados simulados.

\section{RESULTADOS E DISCUSSÕES}

Com o objetivo de validar o modelo multifásico utilizado, foram comparadas as velocidades de mínimo jorro simuladas com aquelas observadas experimentalmente sob as mesmas condições. A Figura 1 a seguir ilustra a metodologia empregada para a determinação da velocidade de mínimo jorro simulada com base na distribuição de fração volumétrica de sólidos.

Sob as condições descritas na Figura 1, a velocidade de $0,34 \mathrm{~m} / \mathrm{s}$ foi estipulada como sendo a velocidade de mínimo jorro simulada, caracterizada por um eminente "rompimento" da superfície de partículas. Esta mesma análise foi feita para as demais condições, como disposto na Tabela 1. 


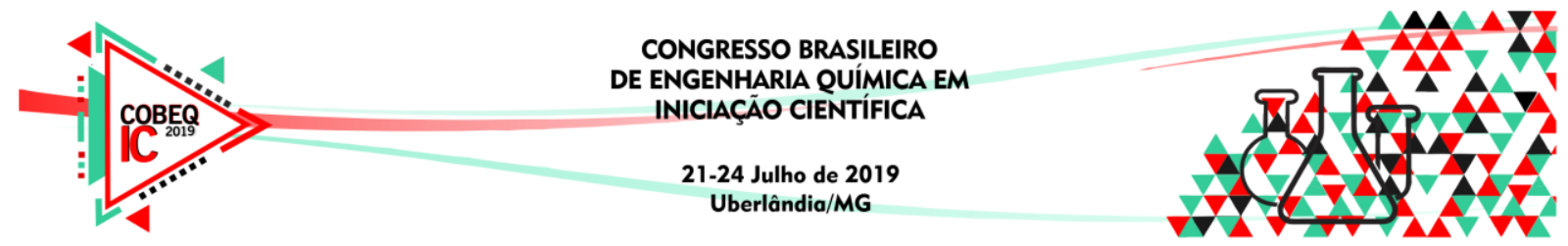

Figura 1 - Distribuição de frações volumétricas de sólidos simuladas nas seguintes velocidades de entrada da água (altura de leito estático de $7 \mathrm{~cm}$; geometria de entrada circular): (a) $0,29 \mathrm{~m} / \mathrm{s}$; (b) $0,34 \mathrm{~m} / \mathrm{s}$; (c) $0,36 \mathrm{~m} / \mathrm{s}$.

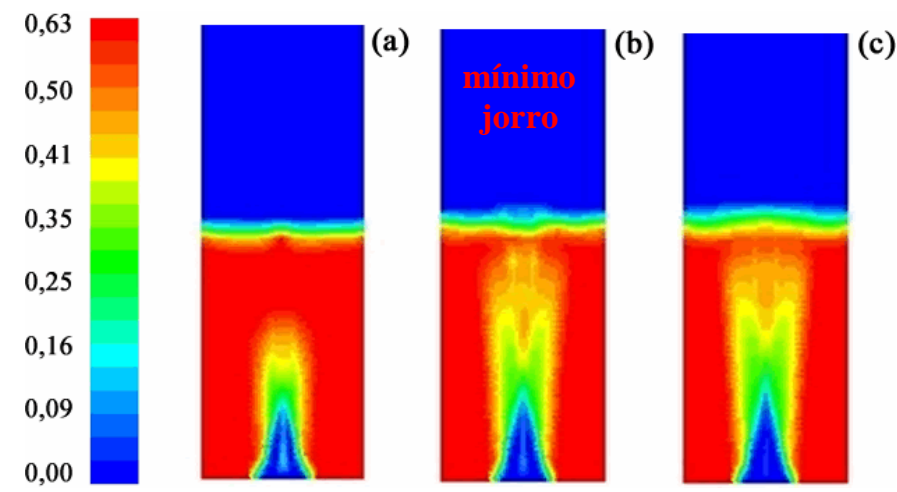

A partir dos dados da Tabela 1, pode-se perceber uma satisfatória concordância entre os dados simulados e experimentais para o leito composto por uma entrada circular, independentemente da altura de leito estático. Por outro lado, para as simulações envolvendo o leito com entrada retangular, observaram-se os maiores desvios com relação aos dados experimentais.

Tabela 1 - Velocidades de mínimo jorro experimental e simulada, juntamente com o desvio percentual, para diferentes condições.

\begin{tabular}{|c|c|c|c|c|}
\hline $\begin{array}{l}\text { Geometria da } \\
\text { Entrada do Leito }\end{array}$ & $\begin{array}{l}\text { Altura de leito } \\
\text { estático }(\mathrm{m})\end{array}$ & $\begin{array}{c}\text { Velocidade de } \\
\text { mínimo jorro } \\
\text { experimental } \\
(\mathrm{m} / \mathrm{s})\end{array}$ & $\begin{array}{c}\text { Velocidade de } \\
\text { mínimo jorro } \\
\text { simulado } \\
(\mathrm{m} / \mathrm{s})\end{array}$ & $\begin{array}{c}\text { Desvio } \\
(\%)\end{array}$ \\
\hline \multirow{2}{*}{ Circular } & 0,07 & 0,366 & 0,340 & $-7,205$ \\
\hline & 0,13 & 0,337 & 0,340 & 1,001 \\
\hline \multirow{2}{*}{ Retangular } & 0,07 & 0,146 & 0,200 & 36,97 \\
\hline & 0,13 & 0,174 & 0,220 & 26,44 \\
\hline
\end{tabular}

Tanto com relação aos dados experimentais, quanto com relação aos resultados numéricos, a mudança da geometria de entrada do leito de jorro, da circular para a retangular (mesmo diâmetro hidráulico), levou a uma diminuição dos valores de velocidade de mínimo jorro, independente da altura de leito estático de material (Tabela 1).

Não foram observadas mudanças significativas nos valores de velocidade de mínimo jorro, experimental e simulada, quando do aumento da altura de leito estático, independe do tipo de geometria da entrada do leito. Foram também comparados os resultados de queda de pressão na condição de mínimo jorro simulados com aqueles obtidos experimentalmente por meio de um manômetro de tubo em "U", cujos valores estão dispostos na Tabela 2 a seguir.

Tabela 2 - Queda de pressão na condição de mínimo jorro experimental e simulada, juntamente com o desvio percentual, para diferentes condições.

\begin{tabular}{|c|c|c|c|c|}
\hline $\begin{array}{c}\text { Geometria de } \\
\text { Entrada do Leito }\end{array}$ & $\begin{array}{c}\text { Altura de leito } \\
\text { estático } \\
(\mathrm{m})\end{array}$ & $\begin{array}{c}\text { Queda de pressão } \\
\text { experimental } \\
(\mathrm{Pa})\end{array}$ & $\begin{array}{c}\text { Queda de pressão } \\
\text { simulada } \\
(\mathrm{Pa})\end{array}$ & $\begin{array}{c}\text { Desvio } \\
(\%)\end{array}$ \\
\hline \multirow{2}{*}{ Circular } & 0,07 & 5036,89 & 5320,86 & 5,64 \\
\cline { 2 - 5 } & 0,13 & 5261,46 & 5834,01 & 10,88 \\
\hline \multirow{2}{*}{ Retangular } & 0,07 & 4959,57 & 5310,88 & 7,08 \\
\cline { 2 - 5 } & 0,13 & 5319,50 & 5882,66 & 10,59 \\
\hline
\end{tabular}




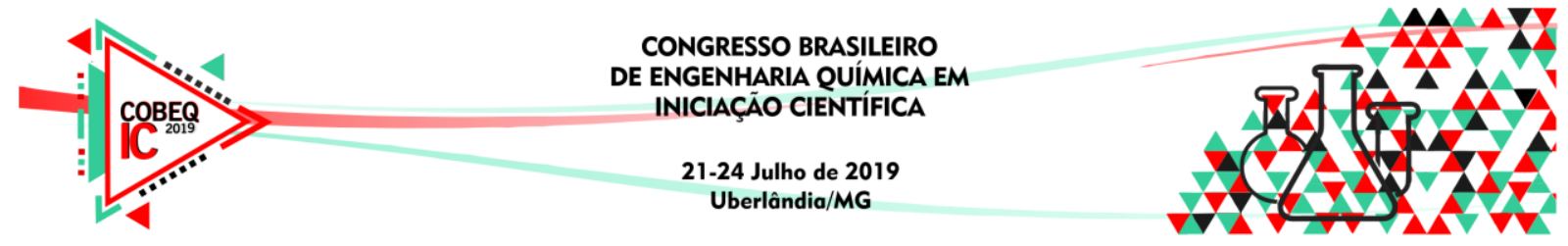

Analisando os desvios obtidos na Tabela 2, pode-se observar, também, uma satisfatória previsão da queda de pressão na condição de mínimo jorro por parte das simulações CFD, tanto para a entrada do leito circular (máximo desvio de 10,9\%), quanto para a entrada retangular (máximo desvio de 10,6\%).

A fim de ilustração, a Figura 2 a seguir, compara as curvas características experimentais e simulada do leito de jorro modificado, para a condição de entrada retangular e altura de leito estático de $0,07 \mathrm{~m}$.

Figura 2 - Curvas características simulada e experimental (altura de leito estático de 0,07 m; geometria de entrada retangular).

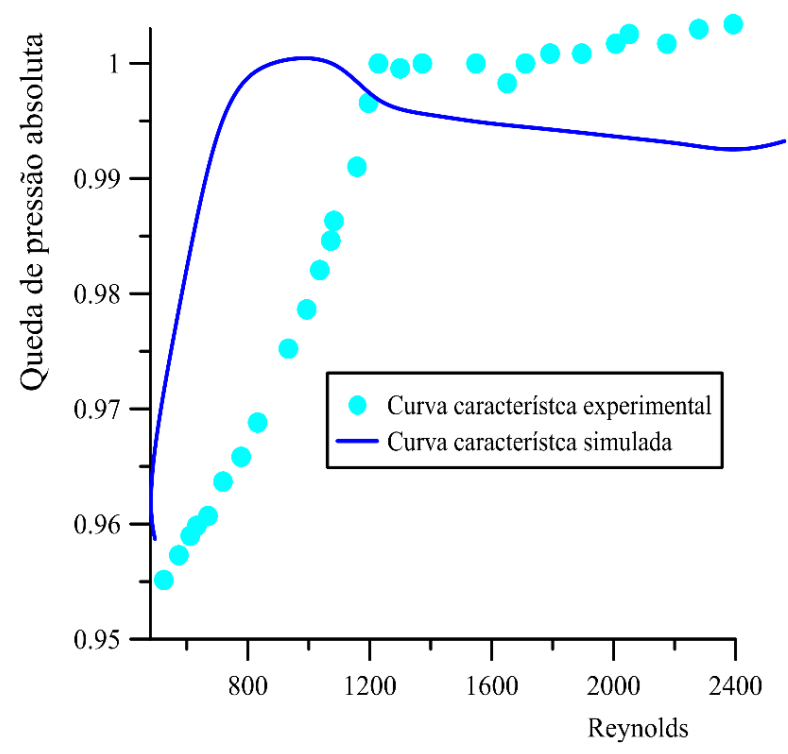

Nota-se, conforme comentado na Tabela 2, uma satisfatória correspondência entre as quedas de pressão de mínimo jorro simulada e experimental. Por outro lado, o valor da velocidade de mínimo jorro determinado por meio da visualização tão somente da curva característica simulada, foi menor do que aquele observado experimentalmente (Figura 2). Isto contradiz com o relatado na Tabela 2, onde o valor de velocidade de mínimo jorro simulado foi determinado por meio da visualização da distribuição de fração de sólidos (baseado na iminência da eclosão da superfície).

Normalmente, utiliza-se apenas a curva característica para se determinar a condição de mínimo jorro experimental. Porém, constatou-se aqui que a simples visualização da curva característica simulada não é suficiente para caracterizar uma condição de mínimo jorro. Isto pode ser visualizado por meio da Figura 3 que mostra a distribuição de fração de sólidos naquela condição que seria de mínimo de jorro visualizada somente pela curva característica da Figura 2. 


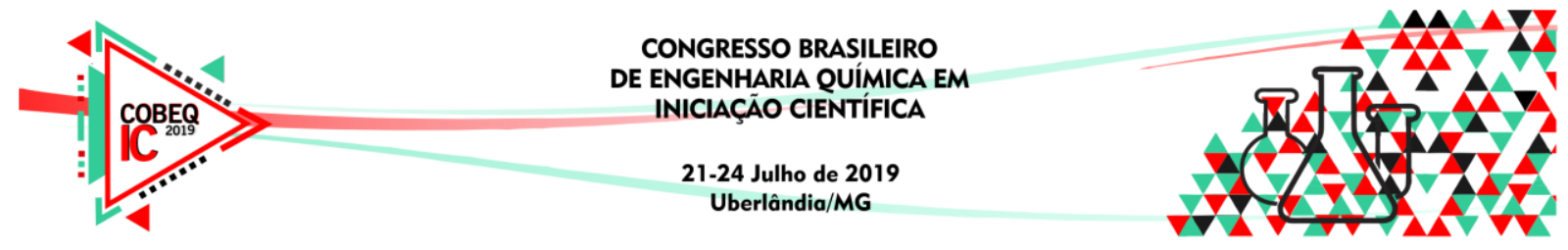

Figura 3 - Distribuição de fração volumétrica de sólidos simulada na condição de mínimo jorro baseada na curva característica da Figura 2.

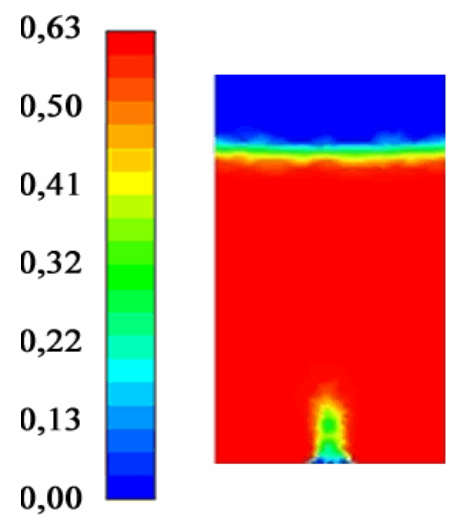

Como pode ser visto na Figura 3, a condição de mínimo jorro dada pela curva característica simulada na Figura 2 não condiz com a característica normalmente descrita do comportamento das partículas, ou seja, não se observou a presença de movimento ou de iminência de eclosão da superfície. Este comportamento foi semelhante para todas as condições mostradas na Tabela 2.

Desta forma, a visualização da distribuição de fração de sólidos simulada é de suma importância para a caracterização do escoamento de partículas no interior do leito de jorro, pois melhor condiz com a condição obtida experimentalmente. Vale ressaltar que as condições de mínimo jorro acima foram determinadas com base na construção de vídeos baseados na fração volumétrica de sólidos, para uma melhor análise da dinâmica das partículas. Para comparar com a técnica gráfica de determinação da velocidade de mínimo jorro, foram construídas as curvas características simuladas para todas as condições mostradas na Tabela 1. A Figura 4 a seguir ilustra a análise realizada através das curvas características para a condição de $0,131 \mathrm{~m}$ de altura de leito estático, tanto para a geometria de entrada do leito circular, quanto para a retangular.

Figura 4 - Curvas características simuladas para a condição de $0,131 \mathrm{~m}$ de altura de leito estático, tanto para a geometria de entrada do leito circular, quanto para a retangular.

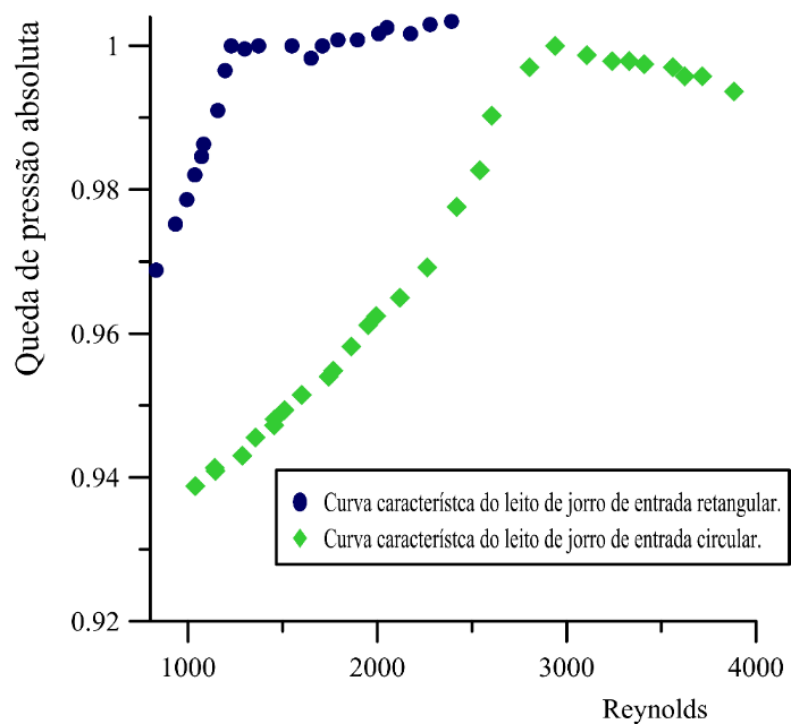




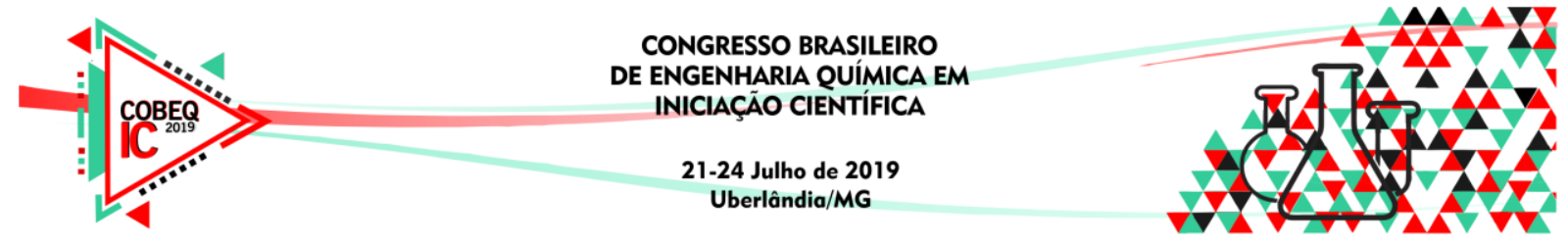

A partir da Figura 4 pode-se verificar, de forma similar àquela observada com a metodologia de visualização da fração volumétrica de sólidos na determinação da condição de mínimo jorro, um aumento no valor da velocidade de mínimo jorro, "deslocamento" do ponto de pressão de mínimo jorro no sentido da esquerda para a direita, quando da modificação da geometria de entrada retangular para a circular, o que condiz com o observado experimentalmente ou com base nas determinações por meio das imagens de distribuição da fração de sólidos simulados.

Apesar do comportamento fisicamente coerente das curvas características, verificou-se neste trabalho que o método de determinação da condição de mínimo jorro simulada através da visualização da distribuição da fração de sólidos melhor condiz com o observado experimentalmente, visto que a determinação das condições de mínimo jorro pela visualização gráfica (curvas características) levou a maiores desvios (máximo de aproximadamente $50 \%$ ).

\section{CONCLUSÕES}

Obteve-se uma satisfatória concordância entre os dados simulados e experimentais para o leito modificado composto por uma entrada circular, independentemente da altura de leito estático. Por outro lado, para as simulações envolvendo o leito com entrada retangular, observaram-se os maiores desvios com relação aos dados experimentais.

Observou-se, também, uma satisfatória previsão da queda de pressão máxima (condição de mínimo jorro) por parte das simulações CFD, tanto para a entrada do leito circular (máximo desvio de 10,9\%), quanto para a entrada retangular (máximo desvio de 10,6\%).

Tanto com relação aos dados experimentais, quanto com relação aos resultados numéricos, a mudança da geometria de entrada do leito de jorro, da circular para a retangular (mesmo diâmetro hidráulico), levou a uma diminuição dos valores de velocidade de mínimo jorro, independente da altura de leito estático de material. O método de determinação da condição de mínimo jorro simulada através da visualização da distribuição da fração de sólidos, em detrimento da metodologia gráfica, melhor condiz com o observado experimentalmente.

\section{REFERÊNCIAS}

DUARTE, C. R. Estudo experimental e de simulação da fluidodinâmica e recobrimento em leito de jorro. Tese de Doutorado, PPGEQ/UFU - Uberlândia/MG, 194p, 2006.

SANTOS, D. A. Contribuições ao estudo da fluidodinâmica em leito de jorro - estudos experimentais e de simulação via CFD. Dissertação de mestrado, PPGEQ/UFU - Uberlândia/MG, 125p, 2011.

MATHUR, K. B.; GISHLER, N.; A Technique for Contacting Gases with Coarse Solid Particles. AIChE Journal, v. 1, p.157-164, 1955. 